\title{
Prostate apoptosis response 4 (PAR4) expression modulates WNT signaling pathways in MCF7 breast cancer cells: A possible mechanism underlying PAR4-mediated docetaxel chemosensitivity
}

\author{
SIMONE APARECIDA DE BESSA GARCIA ${ }^{1,2}$, ANA CAROLINA PAVANELLI ${ }^{1,2}$, \\ NATÁLIA CRUZ E MELO ${ }^{1,2}$ and MARIA APARECIDA NAGAI ${ }^{1,2}$
}

\author{
${ }^{1}$ Discipline of Oncology, Department of Radiology and Oncology, Faculty of Medicine, University of São Paulo, \\ São Paulo, SP 01246-903; ${ }^{2}$ Laboratory of Molecular Genetics, Center for Translational Research in Oncology, \\ Cancer Institute of São Paulo, São Paulo, SP 01246-000, Brazil
}

Received September 26, 2016; Accepted February 8, 2017

DOI: 10.3892/ijmm.2017.2900

\begin{abstract}
Docetaxel is an effective drug for the treatment of metastatic breast cancer. However, the exact mechanisms and/or markers associated with chemosensitivity or resistance to docetaxel remain unclear. We previously showed that the expression of prostate apoptosis response 4 (PAR4) inhibits the growth of MCF7 breast cancer cells and increases their sensitivity to docetaxel. Using cDNA microarray analysis, we evaluated transcriptome changes in MCF7 cells expressing increased levels of PAR4 and control cells before and after docetaxel treatment. Some of the top gene networks generated from the differentially expressed genes were related to the wingless-type MMTV integration 1 (WNT) canonical (WNT/ $\beta$-catenin) and non-canonical ( $\beta$-catenin-independent) pathways. The Human WNT signaling pathway $\mathrm{RT}^{2}$ profiler ${ }^{\mathrm{TM}}$ PCR array was used to validate the effects of PAR4 on the expression pattern of genes involved in the WNT pathway. CACNAD2A3, GDF5 and IL6 were upregulated and NANOG was downregulated in the MCF7 breast cancer cells expressing increased levels of PAR4 after treatment with docetaxel, likely indicating inactivation of the WNT/ $\beta$-catenin pathway. Upregulation of FGF7, LEF1 and TWIST1 indicated activation of the WNT/ $\beta$-catenin pathway. Although preliminary, our findings could be of particular interest for understanding the action of PAR4 in chemosensitivity, particularly to increase the specificity and effectiveness of drug treatment and overcome resistance to chemotherapy. Further studies are needed
\end{abstract}

Correspondence to: Dr Maria Aparecida Nagai, Discipline of Oncology, Department of Radiology and Oncology, Faculty of Medicine, University of São Paulo, Av. Dr. Arnaldo 251, 8th floor, São Paulo, SP 01246-000, Brazil

E-mail: nagai@usp.br

Key words: prostate apoptosis response 4, breast cancer, chemosensitivity, docetaxel, WNT pathways, MCF7 cells to better understand the biological roles of PAR4 in the regulation of WNT pathways in breast cancer cells in response to docetaxel and other chemotherapeutic agents.

\section{Introduction}

Breast cancer is a hormone-dependent disease of complex multifactorial etiology and is the main cause of death in females due to cancer. Approximately 1.15 million new cases of breast cancer are diagnosed every year and it accounts for $14 \%$ of deaths due to cancer in women worldwide (1). The recent trend of a decreased rate of death due to breast cancer is the result of improvements in the early diagnosis of the disease, although the therapeutic options for more advanced stages are still quite limited. Docetaxel, a taxane with antimicrotubule activity, is a very effective drug for the treatment of metastatic breast cancer. The use of adjuvant taxane therapy also offers significant benefits for patients with early stage breast cancer (2). Despite the effectiveness of docetaxel in the treatment of breast cancer, the exact mechanisms and/or markers associated with chemosensitivity or resistance to docetaxel remain unclear (3).

The PKC apoptosis WT1 regulator (PAWR) gene, also called prostate apoptosis response 4 (PAR4), encodes a 342 -amino acid pro-apoptotic protein that plays a central role in cancer cell survival and can be considered a candidate for tumor cell-selective therapy (4). PAR4 protein comprises two nuclear localization signals (NLS): a leucine zipper domain at its carboxy terminal region, and a selective for apoptosis induction in cancer cells (SAC) domain in the central region of the molecule $(5,6)$.

PAR4 plays a role in both the intrinsic and extrinsic apoptotic pathways. Intracellular PAR4 induces apoptosis due to its ability to activate the Fas/FasL/FADD/caspase 8 pathway by inhibiting the $\mathrm{NF}-\kappa \mathrm{B}$ survival pathway, which requires PKA-mediated PAR4 phosphorylation at the T155 residue, and by downregulation of Bcl-2 expression (7-9). On the other hand, PAR4 protein is spontaneously secreted by normal and tumor cells in culture via the classical endoplasmic reticulum (ER)/Golgi pathway, stimulated by stress-inducing 
factors of the ER. The PAR4/GRP78 interaction induces apoptosis via the ER stress pathway and activation of the FADD/caspase-8/caspase-3 pathway (10).

PAR4 is expressed in cells from different tissues, including mammary gland epithelial cells $(11,12)$. Alterations in PAR4 expression have been observed in prostate tumor (13), melanoma (14), renal tumor (15) and hematopoietic (16) cell lines. Decreased PAR4 expression has been demonstrated in different types of cancers, including neuroblastoma (17), endometrial cancer (18), renal cell carcinoma (15), pancreatic tumors (19), and breast cancer $(20,21)$, and PAR4-knockout mice were found to develop spontaneous tumors in various tissues (22).

PAR4 expression is not enough to induce cell death, but it increases the sensitivity of most tumor cells to secondary apoptotic stimulus $(8,23,24)$. Research indicates that PAR4 presents attractive anti-tumoral therapeutic potential, especially due to its tumor cell selectivity. In mouse tumor models, PAR4 overexpression was found to result in tumor regression due to activation of apoptosis via the Fas/FADD receptor (12). Zhao et al (25) also observed that transgenic mice expressing a SAC domain are normal regarding their development and life expectancy in addition to being resistant to tumor growth. In a study on K562 myeloid (Bcr-Abl-positive) cells, PAR4 overexpression enhanced sensitivity to imatinib and inhibitors of histone deacetylases (HDACs), increasing the apoptosis rate of cells resistant to conventional treatment with doxorubicin and agonist Trail and Fas (16). Increased PAR4 expression also increased apoptosis induction in response to ER stress agents in CAKi renal tumor cells through decreased XIAP anti-apoptotic protein levels and p-Akt kinase (26). An in vivo study by Kline et al (27) demonstrated the possible role of PAR4 in colon cancer treatment. Mice receiving nanoliposomes containing the PAR4 expression vector were more susceptible to 5-fluorouracil (5-FU) chemotherapy. Wang et al (28) also showed that PAR4 sensitized colon cancer cells to 5-FU inhibiting NF- $\kappa \mathrm{B}$ and deregulating the miRNA pathway. Increased expression of PAR3 in SW480 and HT29 tumor cells led to interaction between PAR4 and NF- $\mathrm{BB}$ in the cytoplasm, avoided nuclear translocation of p65 and p50 subunits, and reduced cell survival. Furthermore, cells with increased PAR4 expressed reduced levels of DROSHA, a regulator protein in the miRNA pathway, leading to increased pro-apoptotic (Bim) target translation and decreased translation of anti-apoptotic targets, such as Bcl-2 (28).

In rats, Alvarez et al (29) demonstrated that treatment of mammary tumors with adriamycin and cyclophosphamide, followed by paclitaxel, led to significant tumor regression and that the tumors that relapsed after chemotherapy exhibited markedly reduced PAR4 expression. The same was found for patients with breast cancer; PAR4 levels were associated with increased recurrence over 5 years compared to women with breast tumors expressing higher levels of PAR4 (29). Previous results from our group indicated that the expression of PAR4 modulates proliferation and cell death and affects the response of MCF7 breast cancer cells to treatment with docetaxel (23). However, the possible mechanisms involved in the PAR4-mediated chemosensitivity to docetaxel remain largely unknown. In the present study, we provide evidence that PAR4 modulates several genes involved in the wingless-type
MMTV integration 1 (WNT) signaling pathway that could take part in docetaxel chemosensitivity in breast cancer cells.

\section{Materials and methods}

Cell line, plasmid transfection, and docetaxel treatment. The MCF7 cell line derived from breast adenocarcinoma is representative of luminal A breast tumors and was obtained from the American Type Culture Collection $\left(\mathrm{ATCC}^{\circledR}\right.$, HTB-22; Manassas, VA, USA). MCF7 cells were stably transfected with $p c$ CMV6-XL6-PAR4 plasmid expressing the full-length PAR4 cDNA, named MCF7pcPAR4 or with empty vector $p c$ CMV6-XL6-NEO, named MCF7 $p c$ NEO (OriGene Technologies, Inc., Rockville, MD, USA). Transfection was performed using TurboFectin 8.0 (OriGene Technologies, Inc.) according to the manufacturer's recommendations. Twenty-four hours after transfection, the cells were selected with geneticin (Gibco, Gaithersburg, MD, USA). The selected clones were screened for PAR4 mRNA and protein expression by real-time PCR and western blotting, respectively. MCF $7 p c$ NEO and MCF7pcPAR4 cells were treated with $100 \mathrm{nM}$ docetaxel (BD Pharmingen, San Jose, CA, USA) for $24 \mathrm{~h}$ for the cDNA microarray analysis or $50 \mathrm{nM}$ docetaxel for $24 \mathrm{~h}$ for the PCR array analysis. The MCF7pcNEO and MCF7pcPAR4 control cells were maintained with the vehicle solution (absolute ethanol) for $24 \mathrm{~h}$.

RNA extraction. Before and after docetaxel treatment for $24 \mathrm{~h}$, total RNA was extracted from the MCF7pcNEO and MCF7pcPAR4 cells using the acid guanidinium thiocyanate-phenol-chloroform method (30). The RNA concentration was determined using a NanoDrop ND-1000 spectrophotometer (Thermo Fisher Scientific, Waltham, MA, USA). The sample integrity was also determined by RNA Nano Chips using the Agilent 2100 Bioanalyzer platform (Agilent Technologies, Inc., Santa Clara, CA, USA). High quality RNA was purified using the RNeasy Mini kit (Qiagen, Germantown, MD, USA).

cDNA microarray analysis. The cDNA microarray analysis was performed using the Low Input RNA Linear Amplification kit 2-color (GE Healthcare Bio-Sciences, Piscataway, NJ, USA) for the amplification of $200 \mathrm{ng}$ of purified RNA. T7 RNA polymerase was added to generate complementary RNA (cRNA) and the test samples were labeled with cyanine 5 (Cy-5) fluorochrome. The reference sample was labeled with cyanine 3 (Cy-3) fluorochrome. The cRNAs were purified using the RNeasy Mini kit (Qiagen, Germantown, MD, USA), and $825 \mathrm{ng}$ of labeled cRNAs were hybridized with the Human GE $4 \mathrm{x} 44 \mathrm{~K}$ V2 slides (Agilent Technologies, Inc.) for $17 \mathrm{~h}$ at $65^{\circ} \mathrm{C}$. The slides were washed with GE Wash Buffer 1 and GE Wash Buffer 2 (Agilent Technologies, Inc.) following the manufacturer's instructions and digitized by the Agilent Microarrays Scanner (Agilent Technologies, Inc.). The data were quantified and the quality control was performed using Agilent FE Software (Agilent Technologies, Inc.). The extracted data were imported to Agilent Gene Spring 12.5-GX Analyze Program (Agilent Technologies, Inc.). The IPA Ingenuity ${ }^{\circledR}$ system (Qiagen) was used to identify and construct gene networks.

The data discussed in this publication have been deposited in NCBI's gene Expression Omnibus (31) and are accessible 
through GEO Series accession number GSE81064 (http://www. ncbi.nlm.nih.gov/geo/query/acc.cgi?acc=GSE81064).

Human WNT signaling pathway $R T^{2}$ profiler ${ }^{T M}$ PCR array. The Human WNT signaling pathway $\mathrm{RT}^{2}$ profiler ${ }^{\mathrm{TM}} \mathrm{PCR}$ array plate is a panel containing 84 key genes involved in the canonical and non-canonical WNT pathway and 5 endogenous genes for reaction control. The reaction was performed according to the manufacturer's instructions except for the cDNA synthesis, which was carried out using the High capacity archive kit (Applied Biosystems Life Technologies, Foster City, CA, USA). Real-time PCR was performed using SYBR-Green Master Mix (Qiagen) and processed in the GeneAmp 5700 Sequence Detection system (Applied Biosystems Life Technologies, Singapore). The data were exported to and analyzed by $\mathrm{RT}^{2}$ profiler PCR array data analysis template v 3.3 (SABiosciences, Germantown, MD, USA).

Statistical analysis. Gene expression data derived by Agilent was compared for MCF7pcPAR4 vs. MCF7pcNEO cells before and after treatment with docetaxel. According to our experimental design, the statistical test selected was two-way ANOVA. The P-value computation algorithm and the P-value correction test applied were asymptotic and Benjamini Hochberg FDR, respectively. A Venn diagram appears when two-way ANOVA tests are performed and reflects the union and intersection of entities passing the chosen cut-off. For Gene Ontology (GO) analysis the P-value cut-off was set at 1.0. All tests were two-sided and an adjusted $\mathrm{P}<0.05$ was considered to indicate a statistically significant difference.

\section{Results}

In a previous study, we showed that PAR4 upregulation increases the sensitivity of MCF7 breast cancer cells to docetaxel (23). MCF7 cells transfected with the expression vector for PAR4 (MCF7pcPAR4) exhibited an $\sim 4.5$-fold increase in PAR4 protein expression in comparison with the level noted in the MCF7pcNEO cells, which were transfected with the empty vector (Figs. 1A and B). After treatment with docetaxel, the MCF7 cells with increased PAR4 expression exhibited increased proliferation restraint when compared with the MCF7pcNEO cells. Similar effects on cell proliferation were observed following treatment with docetaxel at 50 and $100 \mathrm{nM}$ for $24 \mathrm{~h}$ (Fig. 1C). To better understand the mechanisms involved in PAR4-mediated chemosensitivity, we evaluated the effect of PAR4 on the expression profile of MCF7 breast cancer cells before and after treatment with docetaxel. A Venn diagram was used to represent the number of differentially expressed genes that were common or exclusive in the different comparisons (Fig. 2).

Before docetaxel treatment, 1,045 genes were upregulated and 549 were downregulated in the MCF7pcPAR4 cells vs. MCF $7 p c$ NEO cells, with 702 of the upregulated genes and 433 of the downregulated genes found exclusively in this comparison. After docetaxel treatment, a total of 355 genes were upregulated and 178 were downregulated in the MCF7 $p c$ PAR4 cells vs. MCF7 $p c$ NEO cells, with 275 of the upregulated genes and 147 of the downregulated genes found exclusively in this comparison. Forty-one of the upregulated
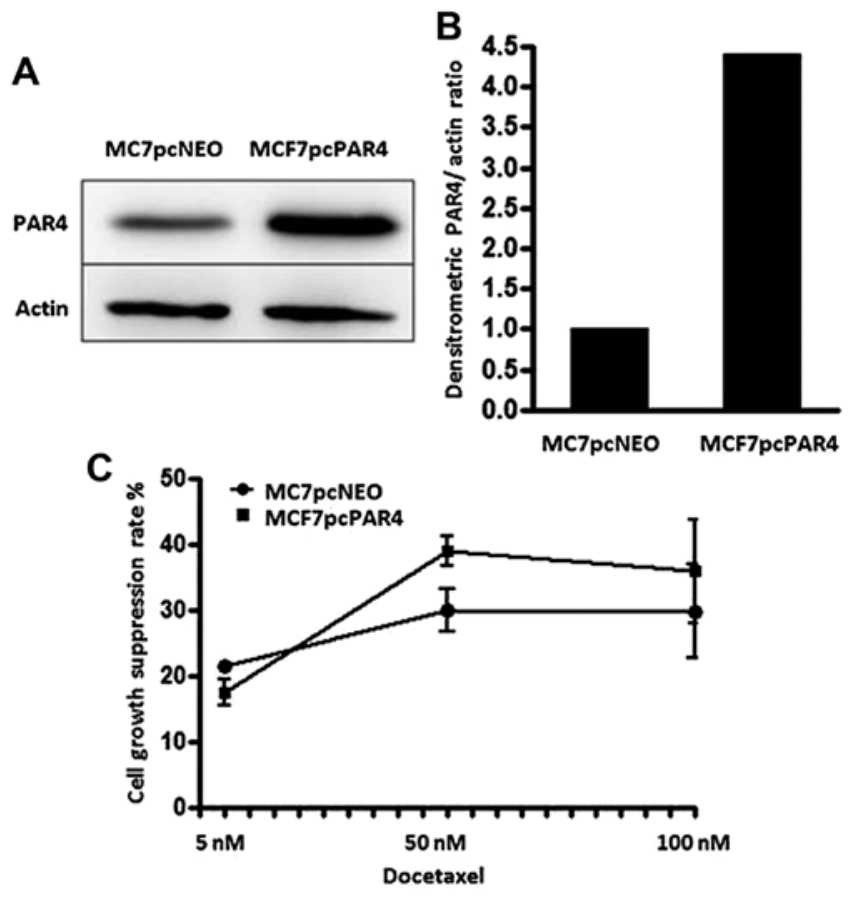

Figure 1. Analysis of PAR4 transfection efficiency and the effect of docetaxel treatment on MCF7 cells. (A) Transfection efficiency analysis was determined by western blotting. (B) Graphic representation of western blotting densitometric analysis using ImageJ software. (C) Effects of docetaxel on the growth suppression rate after treatment for $24 \mathrm{~h}$ in MCF7pcNEO and MCF7pcPAR4 cells using the CyQUANT ${ }^{\circledR}$ Cell Proliferation Assays (Thermo Fisher Scientific, Waltham, MA, USA).

genes were identified both before and after docetaxel treatment, but only 6 downregulated genes were identified both before and after docetaxel treatment.

Gene Ontology (GO) analysis was performed to categorize the differentially expressed genes in terms of the cellular processes in which they participate. Considering the upregulated genes, cell communication, extracellular structure organization and cellular developmental processes were the top categories in the comparisons of MCF7pcPAR4 and MCF $7 p c$ NEO cells before and after docetaxel treatment. Regardless of the observed enrichment, the number of genes represented varied by $\geq 50 \%$ in some categories after treatment with docetaxel. Among the categories we highlight secretion by cell, signal transduction by phosphorylation, cell cycle, cell death, cell cycle processes, microtubule-based processes, and execution phase of apoptosis, which are related to the pro-apoptotic function of PAR4 and anti-microtubule and anti-mitotic actions of docetaxel (Fig. 3).

To assess the possible interactions between the differentially expressed genes, numerous gene networks were generated using the IPA Ingenuity ${ }^{\circledR}$ program for the comparisons between MCF7pcPAR4 and MCF7pcNEO cells before and after treatment with docetaxel. Many of the gene networks generated revealed the modulation of several genes directly or indirectly related to the WNT signaling pathway, such as WNT1, WNT7A, WNT8A, WNT9A, FZD8, TCF, LEF1, SOX5, SOX7, SOX11, SOX17, WISP2 and RUNX2 (Figs. 4-6). We observed an inversion in the expression patterns of some genes related to the WNT pathway after docetaxel treatment, including $W N T 1$, which was upregulated in the MCF7pcPAR4 


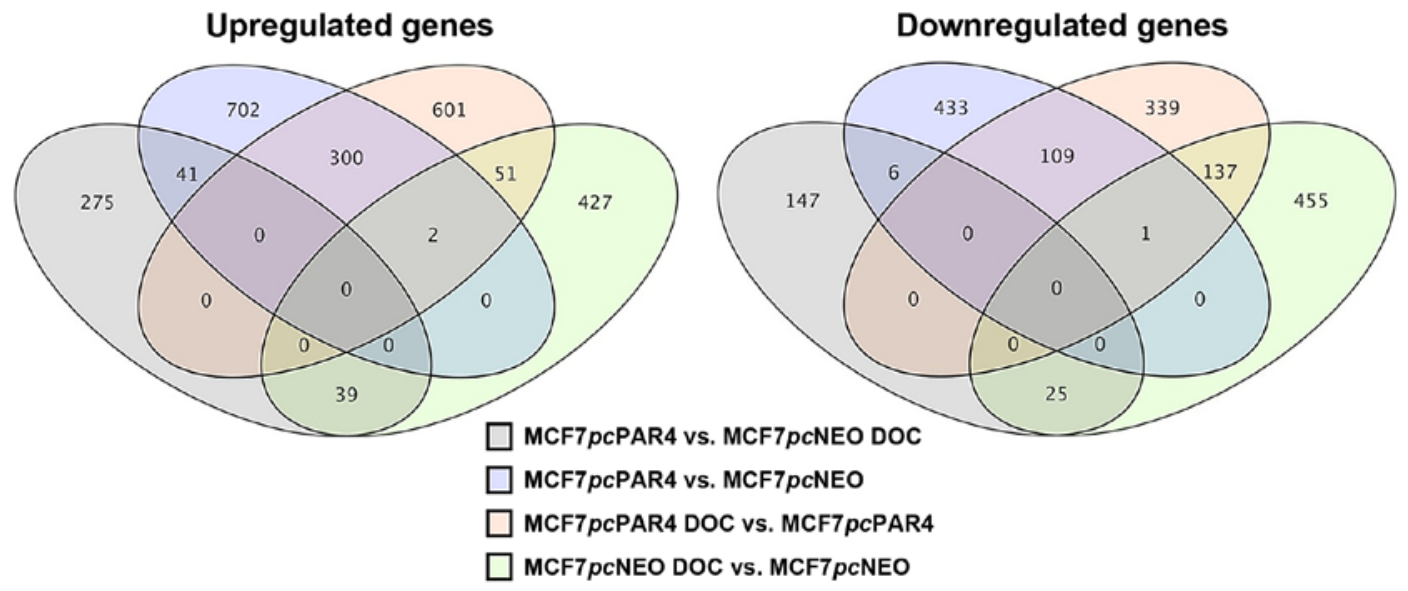

Figure 2. Venn diagrams of the gene distribution between MCF7pcPAR4 and MCF7pcNEO cells before and after docetaxel treatment. DOC, cells treated with docetaxel.
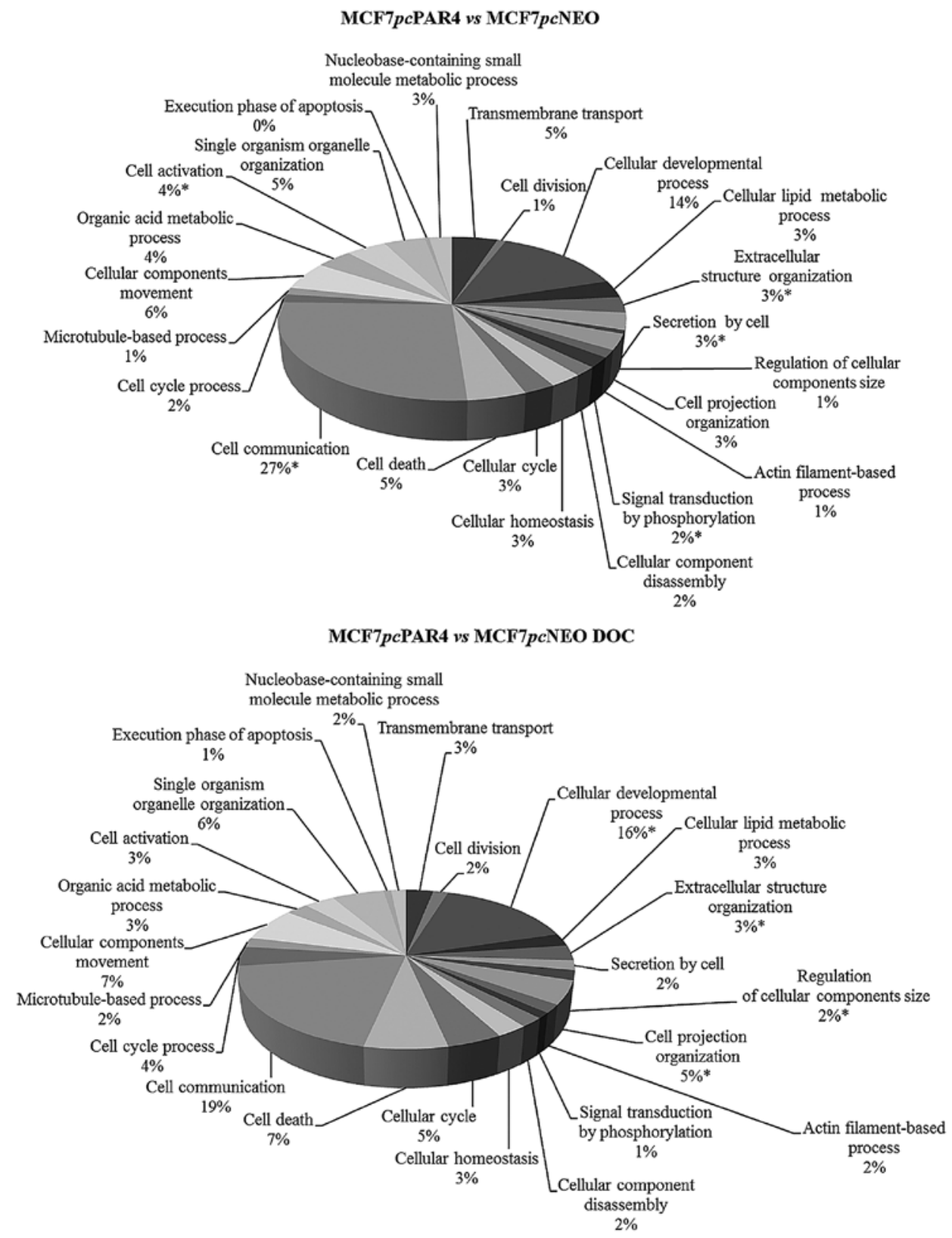

Figure 3. Distribution of Gene Ontology categories of the upregulated genes in MCF7pcPAR4 and MCF7pcNEO cells before and after docetaxel treatment. DOC, cells treated with docetaxel. "Significantly enriched categories. 


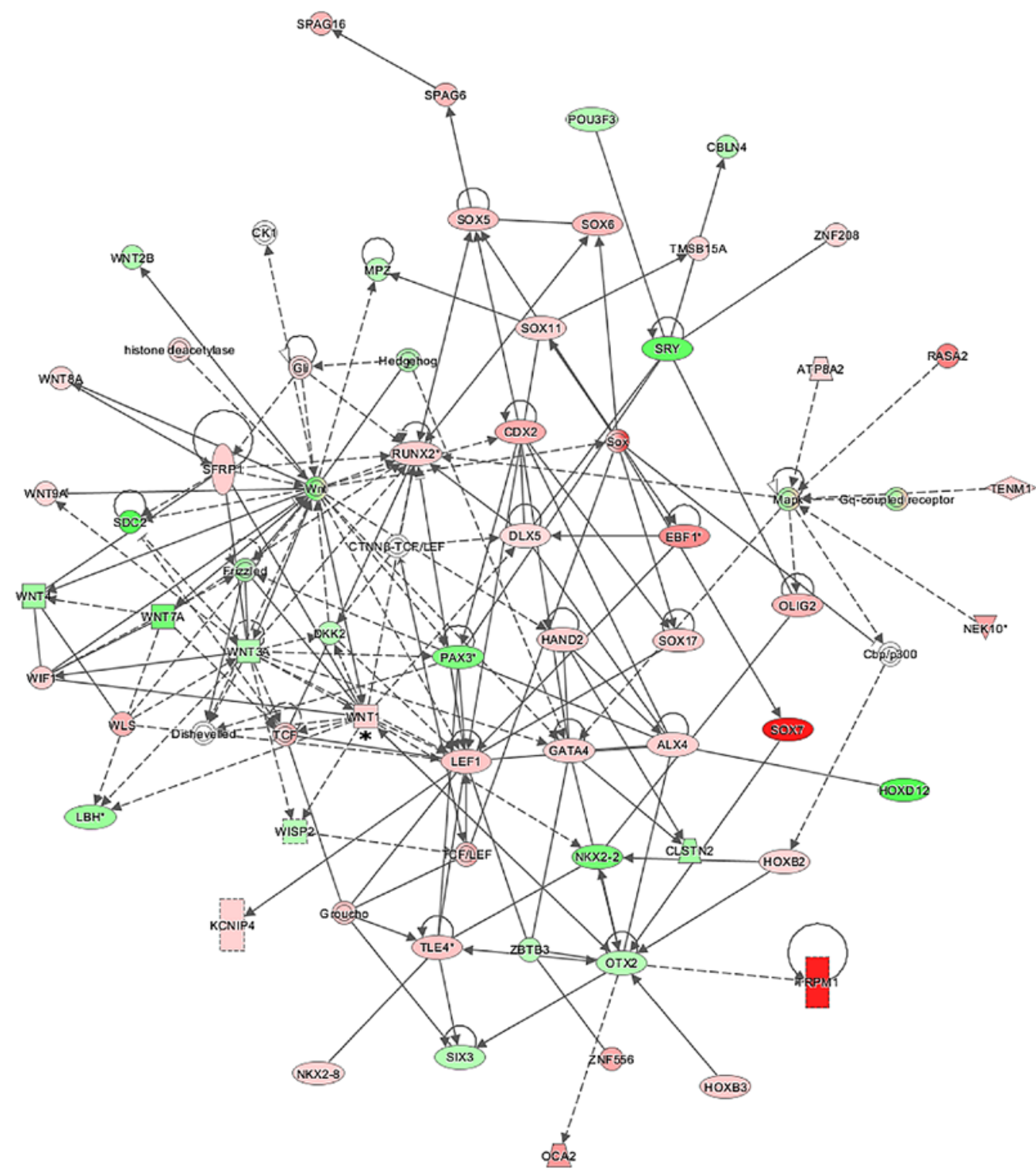

Figure 4. Molecular interaction network of the upregulated and downregulated genes in the MCF7pcPAR4 vs. MCF7pcNEO cell comparison showing the upregulation of WNT1 expression before docetaxel treatment. Genes or gene products are represented as nodes, and the biological relationship between two nodes is represented as an edge. The intensity of the node color indicates the degree of upregulation (red) or downregulation (green). Edges are displayed with various labels that describe the nature of the relationship between the nodes: - , binding only; $\rightarrow$, acts on; $ง$, auto-regulation; *, WNT1 gene upregulation. Dotted edges represent an indirect interaction.

cells before treatment (Fig. 4) but downregulated after treatment with docetaxel (Fig. 5), and members of the Frizzled gene family, which were found to be downregulated in the MCF7pcPAR4 cells before treatment and upregulated after docetaxel treatment (Fig. 6).

In order to validate the effects of PAR4 expression on the regulation of genes involved in the WNT signaling pathway in our experimental model, we used the human WNT signaling pathway $\mathrm{RT}^{2}$ profiler ${ }^{\mathrm{TM}}$ PCR array. Fig. 7 shows the expression profile of 84 genes involved in the WNT signaling pathway in $\mathrm{MCF} 7 p c \mathrm{PAR} 4$ vs. MCF7p $\mathrm{NEO}$ cells before and after treatment with docetaxel. Four transcripts (CDON, CTGF, TCF4 and $W N T 3 A$ ) were regulated by increased PAR4 expression and exhibited no significant modulation after docetaxel treatment. Seven transcripts (CACNA2D3, DAB2, GJA1, LEF1, NANOG, PDGFRA and WNT9A) were modulated in MCF7 cells expressing increased levels of PAR4 only in the presence of docetaxel. Seventeen transcripts $(A H R, C D H 1, C D K N 2 A$, DKK1, EGR1, FGF7, FST, GDF5, IL6, NRCAM, NTRK2, RUNX2, SOX2, SOX9, T, TWIST1 and WISP1) were regulated by increased PAR4 expression and further modulated in opposite or incremental directions by docetaxel treatment.

\section{Discussion}

Previous data from our group showed that PAR4 inhibits the growth of breast cancer cells and increases their sensitivity to docetaxel (23). In the present study, to identify differentially expressed genes potentially involved in PAR4-mediated chemosensitivity to docetaxel, we evaluated the expression profiles of MCF7 breast cancer cells expressing different levels of PAR4, before and after docetaxel exposure. 


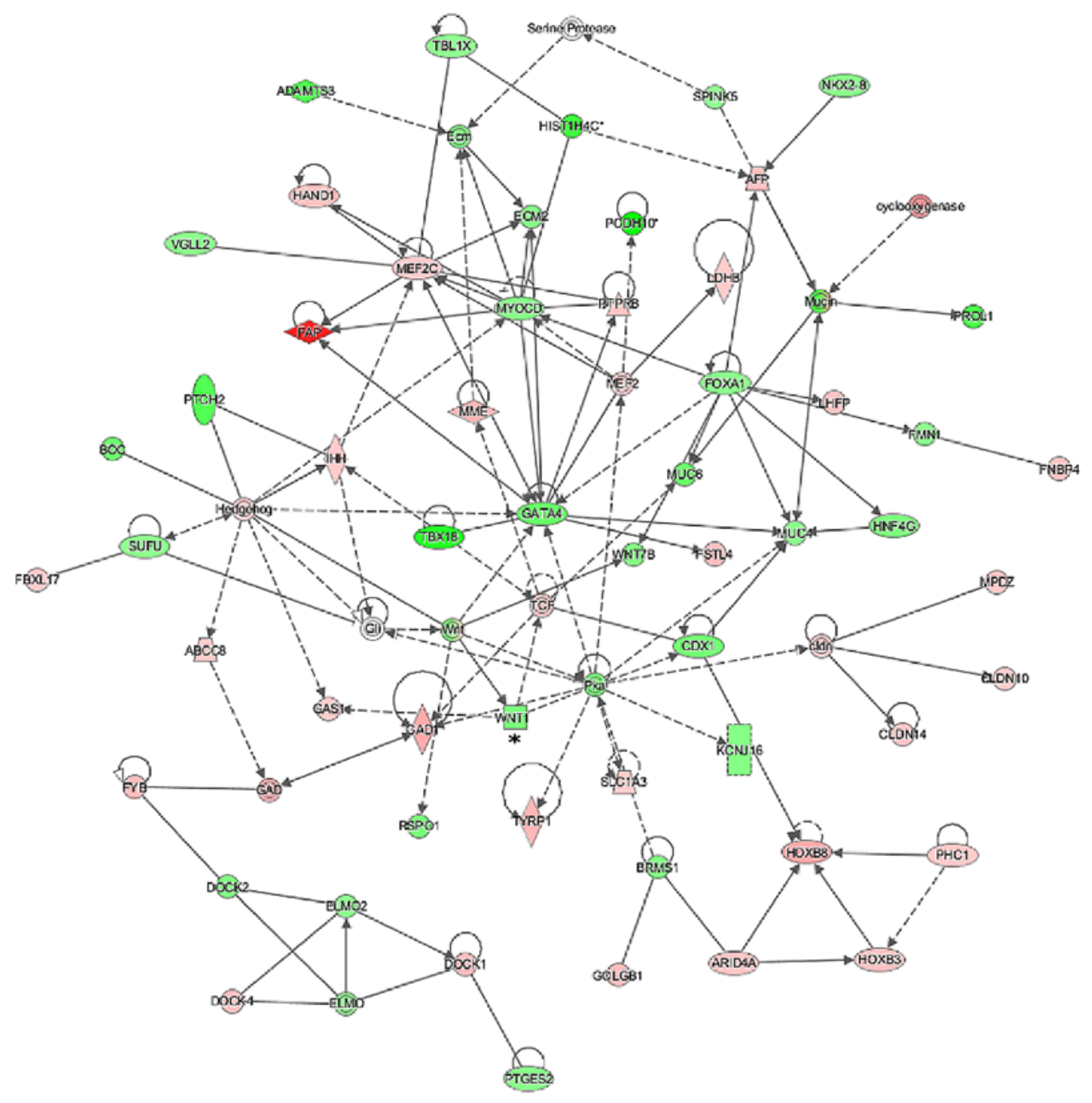

Figure 5. Molecular interaction network of the upregulated and downregulated genes in the MCF7pcPAR4 vs. MCF7pcNEO cell comparison showing the downregulation of WNT1 expression after docetaxel treatment. Genes or gene products are represented as nodes, and the biological relationship between two nodes is represented as an edge. The intensity of the node color indicates the degree of upregulation (red) or downregulation (green). Edges are displayed with various labels that describe the nature of the relationship between the nodes: - , binding only; $\rightarrow$, acts on; 5 , auto-regulation; *, WNT1 gene downregulation Dotted edges represent an indirect interaction.

Notably, the GO analysis of the differentially expressed genes revealed that some of the cellular processes modulated by PAR4 overexpression in the presence and absence of docetaxel are related to the pro-apoptotic functions of PAR4 and anti-microtubule and anti-proliferative actions of docetaxel (e.g., cell division, cell cycle processes, microtubule-based processes and execution phase of apoptosis), confirming the functionality of our experimental model.

Using the IPA Ingenuity ${ }^{\circledast}$ program, several gene networks were generated from the differentially expressed genes. Many of the top gene networks generated were related to the canonical and non-canonical WNT signaling pathway. The WNT signaling pathway is highly conserved and can be divided into two signaling pathways: the $\mathrm{WNT} / \beta$-catenin pathway referred to as the canonical pathway and the $\beta$-catenin-independent WNT signaling referred to as the non-canonical pathway. The WNT signaling pathways control important biological processes, such as embryonic development, proliferation, cell migration, cell polarity, morphogenesis and tissue regeneration, and alterations in this signaling pathway have been shown to be involved in the tumorigenic process of a number of malignancies, including breast cancer $(32,33)$.

WNT1 and members of the Frizzled family of genes, such as FZD8, were upregulated and downregulated, respectively, in cells expressing increased levels of PAR4 before docetaxel treatment and were inversely modulated after docetaxel treatment. The FZDs are transmembrane cell surface receptors activated by the WNT family of lipoglycoproteins to activate canonical and/or non-canonical WNT signaling (34). In invasive breast tumors, WNT1 expression seems to promote differentiation and apoptosis but inhibits proliferation, and is associated with a better prognosis in a subgroup of patients with stage II disease (35). FZD8 was shown to be overexpressed in human lung cancer tissue and cell lines. Furthermore, FZD8 knockdown by siRNA has been shown to inhibit cell proliferation, decrease the activity of the WNT pathway in in vitrolin vivo models, and sensitize lung cancer cells to docetaxel chemotherapy (36). In breast cancer, inhibition of FZD8 expression in CRL2335 cells in the presence of cisplatin plus TRAIL was found to reduce 
A

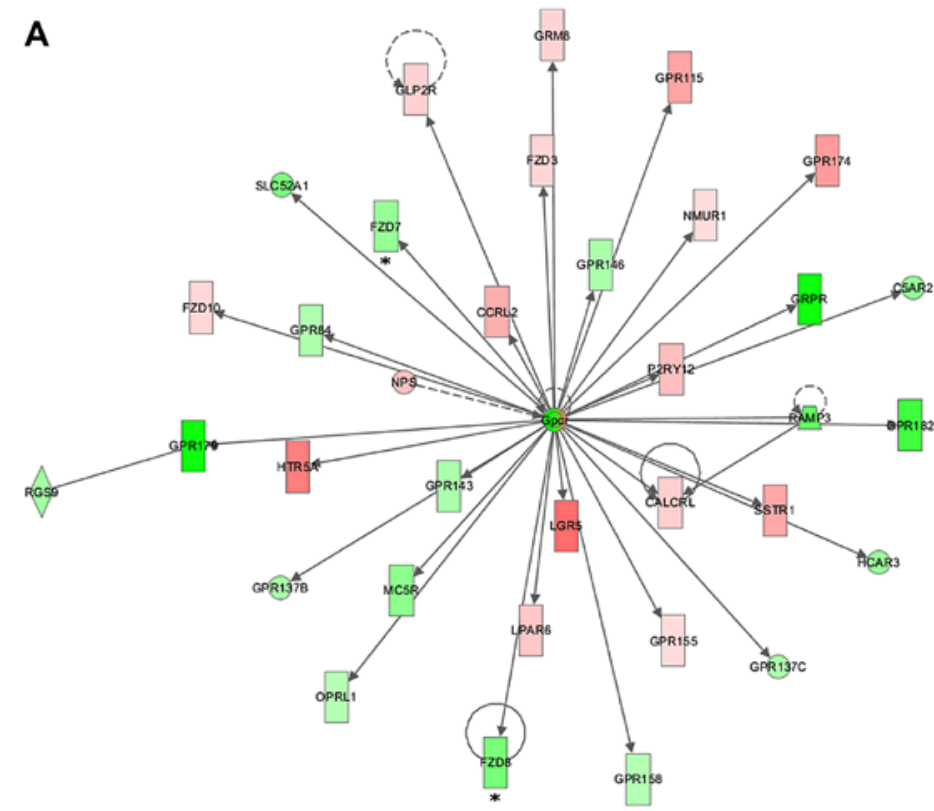

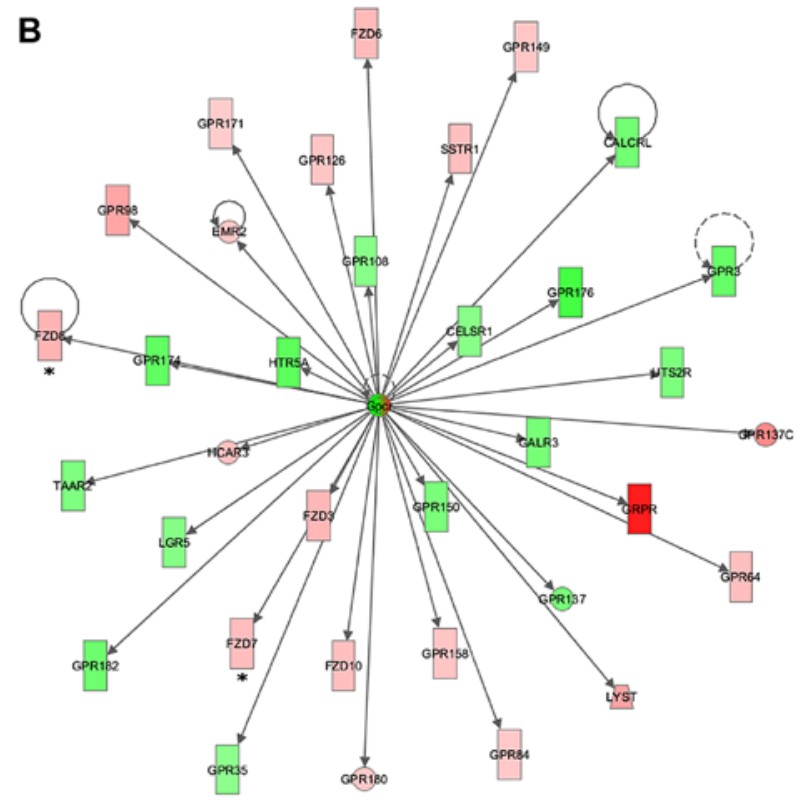

Figure 6. Molecular interaction network of the upregulated and downregulated genes in the MCF7pcPAR4 vs. MCF7pcNEO cell comparison showing the inversion of the FZD7 and FZD8 expression pattern after docetaxel treatment. Upregulated (red) and downregulated (green) genes in the MCF7pcPAR4 and MCF7p $c$ NEO cells in the (A) absence and (B) presence of docetaxel. Genes or gene products are represented as nodes, and the biological relationship between two nodes is represented as an edge. The intensity of the node color indicates the degree of upregulation (red) or downregulation (green). Edges are displayed with various labels that describe the nature of the relationship between the nodes: - , binding only; $\rightarrow$, acts on, $\lrcorner$, auto-regulation, *, FZD genes with expression patterns modified in the absence and presence of docetaxel. Dotted edges represent an indirect interaction. (A) MCF7pcPAR4 vs. MCF7 $p c$ NEO cells before docetaxel treatment. (B) MCF7pcPAR4 vs. MCF7pcNEO cells after docetaxel treatment.

$\beta$-catenin and survivin levels, leading to increased apoptosis. FZD8 expression was also observed in residual triple-negative breast tumors treated with cisplatin and TRAIL, suggesting that FZD8 expression may play a role in chemoresistance in triple-negative breast tumors (37). Taken together, the data suggest that the modulation of WNTI and FZD 8 by PAR4 may be one of the mechanisms involved in PAR4-mediated chemosensitivity. Although some attempts have been made to classify the canonical and non-canonical WNT pathways according to the activated WNT receptors or co-receptors, to what extent these pathways may overlap is still controversial. In addition, as WNT signaling is implicated in various diseases, efforts are being made to identify molecules that interfere with the pathway in a cell type-specific manner (38).

We used the human WNT signaling pathway $\mathrm{RT}^{2}$ profiler $^{\mathrm{TM}}$ PCR array to evaluate the effects of PAR4 on the expression of a number of genes involved in the canonical and non-canonical WNT signaling pathways in our experimental model. The expression profiles of EGR1, IL6, SOX2 and WISP1 in MCF7 cells with increased PAR4 expression before treatment with docetaxel likely indicate inactivation of the $\mathrm{WNT} / \beta$-catenin signaling pathway (39-42). However, the expression profiles of CDKN2A, FGF7, TWIST, NTRK2 and SOX9 in the same cell conditions, likely suggest activation of the WNT/ $\beta$-catenin signaling pathway (43-47). Furthermore, the observed expression profiles of CACNAD2A3, GDF5, IL6 and NANOG in MCF7 cells with increased PAR4 expression after treatment with docetaxel suggest inactivation of the WNT/ $\beta$-catenin pathway (41,48-50), whereas the expression of FGF7, TWIST and $L E F 1$ transcripts, under the same cell conditions, suggests that $\mathrm{WNT} / \beta$-catenin signaling is active. These are target genes of the canonical pathway and have been associated with epithelial-mesenchymal transition (EMT) and chemoresistance $(44,51,52)$.

Alterations in the WNT/ $\beta$-catenin signaling pathway have been shown to be a frequent event in the tumorigenic process and chemoresistance in various types of cancer $(53,54)$. In breast cancer, alterations in the WNT/ $\beta$-catenin signaling pathway are critical to tumor development and progression (55). In addition, increased expression of the Frizzled receptors or aberrant activation of $\beta$-catenin have been associated with resistance to radiotherapy and chemotherapy in breast cancer $(56,57)$. Furthermore, downregulation of the WNT/ $\beta$-catenin signaling pathway inhibits EMT and reduces invasion in breast cancer cell lines $(58,59)$. However, to the best of our knowledge no studies have addressed the involvement of PAR4 in the WNT signaling pathway and treatment with docetaxel in breast cancer.

Here we provide evidence that increased PAR4 expression is able to modulate the expression of genes involved in the canonical and non-canonical WNT signaling pathways. Our findings are still preliminary and do not allow us to state that the WNT pathway would be active or inactive in MCF7 cells in the presence of PAR4 with or without treatment with docetaxel, and they do not allow us to predict the exact biological role of these pathways in chemosensitivity to docetaxel in breast cancer cells.

The role of Wnt and its downstream effectors have been demonstrated in primary human breast tumors as well as breast cancer cell lines and are suggested to play an important role in breast tumor development and progression (60-62). Collectively, our study also opens up the possibility to speculate that PAR4 increases breast cancer cell chemosensitivity by the modulation of the WNT signaling pathway. However, investigations using different breast cancer cell lines with 

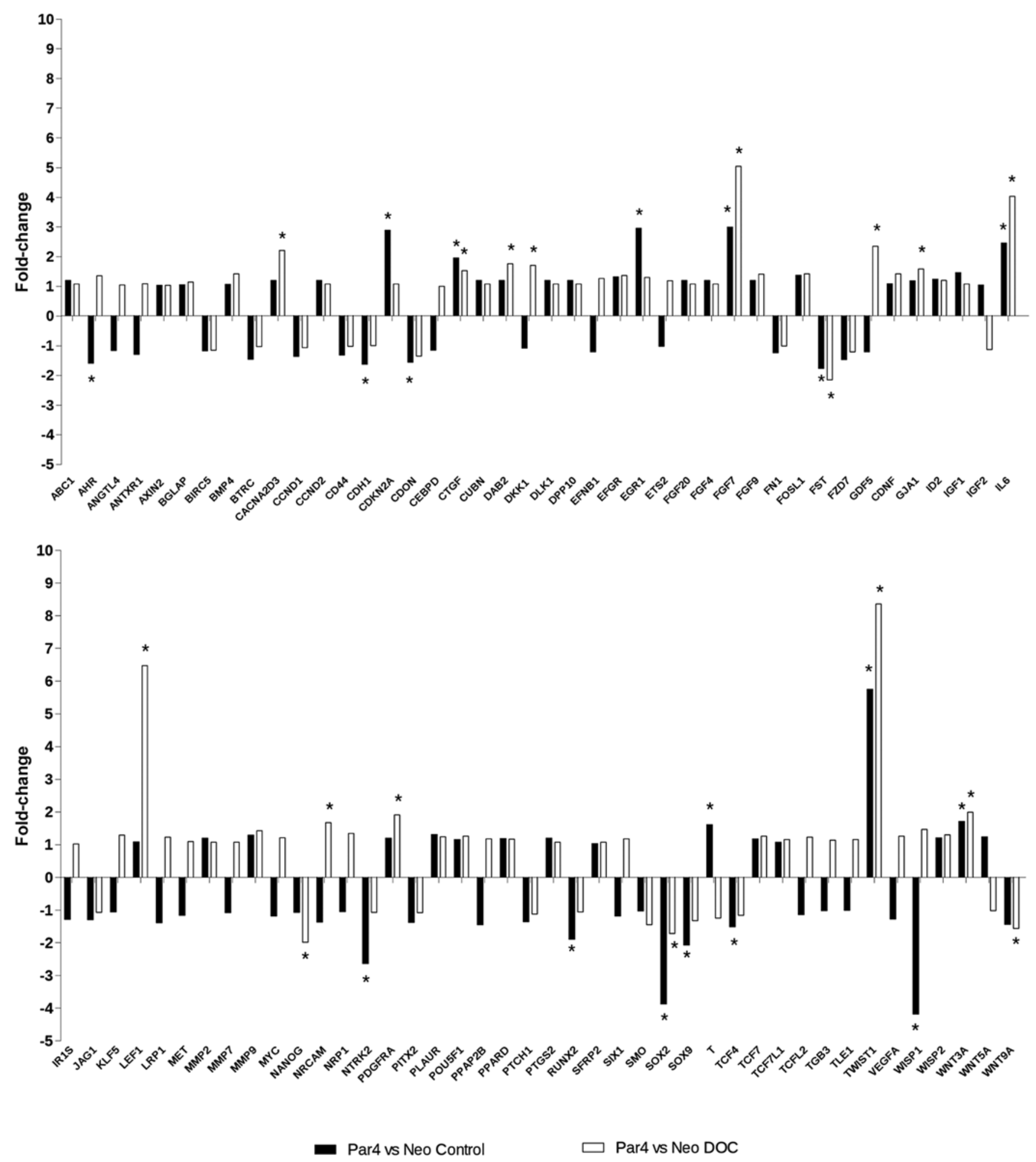

Figure 7. Fold changes in the expression of 84 key genes involved in the WNT pathways by RT ${ }^{2}$ profiler ${ }^{\mathrm{TM}}$ PCR array. *Differentially expressed genes with fold-change $\geq 1.5$.

different expression of PAR4 treated with different chemotherapeutic agents in the presence and absence of WNT pathway inhibitors will need to be performed to better understand the relationship between the expression of PAR4 and the WNT pathway in chemosensitivity to docetaxel and other chemotherapeutic agents; issues that we are currently addressing.

\section{Acknowledgements}

The present study was supported by Fundação de Amparo a Pesquisa do Estado de São Paulo (FAPESP; 2013/07035-4) and Conselho Nacional de Desenvolvimento Científico e Tecnológico (CNPq; 303134/2013-5). 


\section{References}

1. Parkin DM, Bray F, Ferlay J and Pisani P: Global cancer statistics, 2002. CA Cancer J Clin 55: 74-108, 2005.

2. De Laurentiis M, Cancello G, D'Agostino D, Giuliano M, Giordano A, Montagna E, Lauria R, Forestieri V, Esposito A, Silvestro L, et al: Taxane-based combinations as adjuvant chemotherapy of early breast cancer: A meta-analysis of randomized trials. J Clin Oncol 26: 44-53, 2008.

3. Murray CJ, Vos T, Lozano R, Naghavi M, Flaxman AD, Michaud C, Ezzati M, Shibuya K, Salomon JA, Abdalla S, et al: Disability-adjusted life years (DALYs) for 291 diseases and injuries in 21 regions, 1990-2010: a systematic analysis for the Global Burden of Disease Study 2010. Lancet 380: 2197-2223, 2012.

4. Zhao Y and Rangnekar VM: Apoptosis and tumor resistance conferred by Par-4. Cancer Biol Ther 7: 1867-1874, 2008.

5. Sells SF, Han SS, Muthukkumar S, Maddiwar N, Johnstone R, Boghaert E, Gillis D, Liu G, Nair P, Monnig S, et al: Expression and function of the leucine zipper protein Par-4 in apoptosis. Mol Cell Biol 17: 3823-3832, 1997.

6. Hebbar N, Wang C and Rangnekar VM: Mechanisms of apoptosis by the tumor suppressor Par-4. J Cell Physiol 227: 3715-3721, 2012.

7. Boehrer S, Chow KU, Beske F, Kukoc-Zivojnov N, Puccetti E, Ruthardt M, Baum C, Rangnekar VM, Hoelzer D, Mitrou PS, et al: In lymphatic cells par-4 sensitizes to apoptosis by down-regulating bcl-2 and promoting disruption of mitochondrial membrane potential and caspase activation. Cancer Res 62: 1768-1775, 2002.

8. Chakraborty M, Qiu SG, Vasudevan KM and Rangnekar VM: Par-4 drives trafficking and activation of Fas and Fasl to induce prostate cancer cell apoptosis and tumor regression. Cancer Res 61: 7255-7263, 2001.

9. Gurumurthy S, Goswami A, Vasudevan KM and Rangnekar VM: Phosphorylation of Par- 4 by protein kinase A is critical for apoptosis. Mol Cell Biol 25: 1146-1161, 2005.

10. Burikhanov R, Zhao Y, Goswami A, Qiu S, Schwarze SR and Rangnekar VM: The tumor suppressor Par-4 activates an extrinsic pathway for apoptosis. Cell 138: 377-388, 2009.

11. Boghaert ER, Sells SF, Walid AJ, Malone P, Williams NM, Weinstein MH, Strange R and Rangnekar VM: Immunohistochemical analysis of the proapoptotic protein Par-4 in normal rat tissues. Cell Growth Differ 8: 881-890, 1997.

12. Gurumurthy $\mathrm{S}$ and Rangnekar VM: Par- 4 inducible apoptosis in prostate cancer cells. J Cell Biochem 91: 504-512, 2004.

13. Sells SF, Wood DP Jr, Joshi-Barve SS, Muthukumar S, Jacob RJ, Crist SA, Humphreys S and Rangnekar VM: Commonality of the gene programs induced by effectors of apoptosis in androgen-dependent and -independent prostate cells. Cell Growth Differ 5: 457-466, 1994.

14. Lucas T, Pratscher B, Krishnan S, Fink D, Günsberg P, Wolschek M, Wacheck V, Muster T, Romirer I, Wolff K, et al: Differential expression levels of Par-4 in melanoma. Melanoma Res 11: 379-383, 2001

15. Cook J, Krishnan S, Ananth S, Sells SF, Shi Y, Walther MM, Linehan WM, Sukhatme VP, Weinstein MH and Rangnekar VM Decreased expression of the pro-apoptotic protein Par-4 in renal cell carcinoma. Oncogene 18: 1205-1208, 1999.

16. Brieger A, Boehrer S, Schaaf S, Nowak D, Ruthardt M, Kim SZ, Atadja P, Hoelzer D, Mitrou PS, Weidmann E, et al: In bcr-abl-positive myeloid cells resistant to conventional chemotherapeutic agents, expression of Par-4 increases sensitivity to imatinib (STI571) and histone deacetylase-inhibitors. Biochem Pharmacol 68: 85-93, 2004

17. Kögel D, Reimertz C, Mech P, Poppe M, Frühwald MC, Engemann $\mathrm{H}$, Scheidtmann KH and Prehn JH: Dlk/ZIP kinase-induced apoptosis in human medulloblastoma cells: Requirement of the mitochondrial apoptosis pathway. Br J Cancer 85: 1801-1808, 2001.

18. Moreno-Bueno G, Fernandez-Marcos PJ, Collado M, Tendero MJ, Rodriguez-Pinilla SM, Garcia-Cao I, Hardisson D, Diaz-Meco MT, Moscat J, Serrano M, et al: Inactivation of the candidate tumor suppressor par-4 in endometrial cancer. Cancer Res 67: 1927-1934, 2007.

19. Ahmed MM, Sheldon D, Fruitwala MA, Venkatasubbarao K, Lee EY, Gupta S, Wood C, Mohiuddin M and Strodel WE: Downregulation of PAR-4, a pro-apoptotic gene, in pancreatic tumors harboring K-ras mutation. Int J Cancer 122: 63-70, 2008.
20. Nagai MA, Gerhard R, Salaorni S, Fregnani JH, Nonogaki S, Netto MM and Soares FA: Downregulation of the candidate tumor suppressor gene PAR-4 is associated with poor prognosis in breast cancer. Int J Oncol 37: 41-49, 2010.

21. Méndez-López LF, Zapata-Benavides P, Zavala-Pompa A, Aguado-Barrera ME, Pacheco-Calleros J, Rodríguez-Padilla C, Cerda-Flores RM, Cortés-Gutiérrez EI and Dávila-Rodríguez MI: Immunohistochemical analysis of prostate apoptosis response-4 (Par-4) in Mexican women with breast cancer: A preliminary study. Arch Med Res 41: 261-268, 2010.

22. García-Cao I, Duran A, Collado M, Carrascosa MJ, MartínCaballero J, Flores JM, Diaz-Meco MT, Moscat J and Serrano M: Tumour-suppression activity of the proapoptotic regulator Par4. EMBO Rep 6: 577-583, 2005.

23. Pereira MC, de Bessa-Garcia SA, Burikhanov R, Pavanelli AC, Antunes L, Rangnekar VM and Nagai MA: Prostate apoptosis response-4 is involved in the apoptosis response to docetaxel in MCF-7 breast cancer cells. Int J Oncol 43: 531-538, 2013.

24. Jagtap JC, Parveen D, Shah RD, Desai A, Bhosale D, Chugh A, Ranade D, Karnik S, Khedkar B, Mathur A, et al: Secretory prostate apoptosis response (Par)- 4 sensitizes multicellular spheroids (MCS) of glioblastoma multiforme cells to tamoxifen-induced cell death. FEBS Open Bio 5: 8-19, 2014.

25. Zhao Y, Burikhanov R, Qiu S, Lele SM, Jennings CD, Bondada S, Spear B and Rangnekar VM: Cancer resistance in transgenic mice expressing the SAC module of Par-4. Cancer Res 67: 9276-9285, 2007.

26. Lee TJ, Lee JT, Kim SH, Choi YH, Song KS, Park JW and KwonTK: Overexpression of Par-4enhances thapsigargin-induced apoptosis via down-regulation of XIAP and inactivation of Akt in human renal cancer cells. J Cell Biochem 103: 358-368, 2008.

27. Kline CL, Shanmugavelandy SS, Kester M and Irby RB: Delivery of PAR-4 plasmid in vivo via nanoliposomes sensitizes colon tumor cells subcutaneously implanted into nude mice to 5-FU. Cancer Biol Ther 8: 1831-1837, 2009.

28. Wang BD, Kline CL, Pastor DM, Olson TL, Frank B, Luu T, Sharma AK, Robertson G, Weirauch MT, Patierno SR, et al: Prostate apoptosis response protein 4 sensitizes human colon cancer cells to chemotherapeutic 5-FU through mediation of an NF kappaB and microRNA network. Mol Cancer 9: 98, 2010.

29. Alvarez JV, Pan TC, Ruth J, Feng Y, Zhou A, Pant D, Grimley JS Wandless TJ, Demichele A and Chodosh LA; I-SPY 1 TRIAL Investigators: Par-4 downregulation promotes breast cancer recurrence by preventing multinucleation following targeted therapy. Cancer Cell 24: 30-44, 2013.

30. Chomczynski P and Sacchi N: Single-step method of RNA isolation by acid guanidinium thiocyanate-phenol-chloroform extraction. Anal Biochem 162: 156-159, 1987.

31. Edgar R, Domrachev M and Lash AE: Gene Expression Omnibus: NCBI gene expression and hybridization array data repository. Nucleic Acids Res 30: 207-210, 2002.

32. Michaelson JS and Leder P: beta-catenin is a downstream effector of Wnt-mediated tumorigenesis in the mammary gland. Oncogene 20: 5093-5099, 2001.

33. Amin $\mathrm{N}$ and Vincan E: The Wnt signaling pathways and cell adhesion. Front Biosci (Landmark Ed) 17: 784-804, 2012.

34. Dijksterhuis JP, Petersen J and Schulte G: WNT/Frizzled signalling: receptor-ligand selectivity with focus on FZD-G protein signalling and its physiological relevance: IUPHAR Review 3. Br J Pharmacol 171: 1195-1209, 2014.

35. Mylona E, Vamvakaris I, Giannopoulou I, Theohari I, Papadimitriou C, Keramopoulos A and Nakopoulou L: An immunohistochemical evaluation of the proteins Wnt1 and glycogen synthase kinase (GSK)-3 $\beta$ in invasive breast carcinomas. Histopathology 62: 899-907, 2013.

36. Wang HQ, Xu ML, Ma J, Zhang Y and Xie CH: Frizzled-8 as a putative therapeutic target in human lung cancer. Biochem Biophys Res Commun 417: 62-66, 2012.

37. Yin S, Xu L, Bonfil RD, Banerjee S, Sarkar FH, Sethi S and Reddy KB: Tumor-initiating cells and FZD8 play a major role in drug resistance in triple-negative breast cancer. Mol Cancer Ther 12: 491-498, 2013.

38. Niehrs C: The complex world of WNT receptor signalling. Nat Rev Mol Cell Biol 13: 767-779, 2012.

39. Tice DA, Soloviev I and Polakis P: Activation of the Wnt pathway interferes with serum response element-driven transcription of immediate early genes. J Biol Chem 277: 6118-6123, 2002.

40. Ji J, Wei X and Wang Y: Embryonic stem cell markers Sox-2 and OCT4 expression and their correlation with WNT signal pathway in cervical squamous cell carcinoma. Int J Clin Exp Pathol 7: 2470-2476, 2014. 
41. Lam SP, Luk JM, Man K, Ng KT, Cheung CK, Rose-John S and Lo CM: Activation of interleukin-6-induced glycoprotein 130/signal transducer and activator of transcription 3 pathway in mesenchymal stem cells enhances hepatic differentiation, proliferation, and liver regeneration. Liver Transpl 16 1195-1206, 2010.

42. Venkatesan B, Prabhu SD, Venkatachalam K, Mummidi S, Valente AJ, Clark RA, Delafontaine P and Chandrasekar B: WNT1-inducible signaling pathway protein-1 activates diverse cell survival pathways and blocks doxorubicin-induced cardiomyocyte death. Cell Signal 22: 809-820, 2010.

43. Ayyanan A, Civenni G, Ciarloni L, Morel C, Mueller N, Lefort K, Mandinova A, Raffoul W, Fiche M, Dotto GP, et al: Increased Wnt signaling triggers oncogenic conversion of human breast epithelial cells by a Notch-dependent mechanism. Proc Nat Acad Sci USA 103: 3799-3804, 2006.

44. Katoh M and Katoh M: FGF signaling network in the gastrointestinal tract (Review). Int J Oncol 29: 163-168, 2006.

45. Vadnais C, Shooshtarizadeh P, Rajadurai CV, Lesurf R, Hulea L, Davoudi S, Cadieux C, Hallett M, Park M and Nepveu A: Autocrine activation of the Wnt/ $\beta$-catenin pathway by CUX1 and GLIS1 in breast cancers. Biol Open 3: 937-946, 2014.

46. Barreto RA, Walker FR, Dunkley PR, Day TA and Smith DW: Fluoxetine prevents development of an early stress-related molecular signature in the rat infralimbic medial prefrontal cortex. Implications for depression? BMC Neurosci 13: 125, 2012.

47. Akiyama H, Lyons JP, Mori-Akiyama Y, Yang X, Zhang R, Zhang Z, Deng JM, Taketo MM, Nakamura T, Behringer RR, et al Interactions between Sox 9 and beta-catenin control chondrocyte differentiation. Genes Dev 18: 1072-1087, 2004.

48. Wong AM, Kong KL, Chen L, Liu M, Wong AM, Zhu C, Tsang JW and Guan XY: Characterization of CACNA2D3 as a putative tumor suppressor gene in the development and progression of nasopharyngeal carcinoma. Int J Cancer 133: 2284-2295, 2013.

49. Enochson L, Stenberg J, Brittberg $M$ and Lindahl A: GDF5 reduces MMP13 expression in human chondrocytes via DKK1 mediated canonical Wnt signaling inhibition. Osteoarthritis Cartilage 22: 566-577, 2014.

50. Yin D, Tian L, Ye Y, Li K, Wang J, Cheng P, Chen A, Guo F and Huang H: Nanog and $\beta$-catenin: A new convergence point in EpSC proliferation and differentiation. Int J Mol Med 29: 587-592, 2012.

51. Nguyen DX, Chiang AC, Zhang XH, Kim JY, Kris MG, Ladanyi M, Gerald WL and Massagué J: WNT/TCF signaling through LEF1 and HOXB9 mediates lung adenocarcinoma metastasis. Cell 138: 51-62,2009.
52. Je EC, Lca BS and Ga GA: The role of transcription factor TWIST in cancer cells. J Genet Syndr Gene Ther 4: 124, 2013.

53. Loh YN, Hedditch EL, Baker LA, Jary E, Ward RL and Ford CE: The Wnt signalling pathway is upregulated in an in vitro model of acquired tamoxifen resistant breast cancer. BMC Cancer 13: $174,2013$.

54. Ren J, Wang R, Song H, Huang G and Chen L: Secreted frizzled related protein 1 modulates taxane resistance of human lung adenocarcinoma. Mol Med 20: 164-178, 2014.

55. Lamb R, Ablett MP, Spence K, Landberg G, Sims AH and Clarke RB: Wnt pathway activity in breast cancer sub-types and stem-like cells. PLoS One 8: e67811, 2013.

56. Zhang H, Zhang X, Wu X, Li W, Su P, Cheng H, Xiang L, Gao P and Zhou G: Interference of Frizzled 1 (FZD1) reverses multidrug resistance in breast cancer cells through the Wnt $/ \beta$-catenin pathway. Cancer Lett 323: 106-113, 2012.

57. Woodward WA, Chen MS, Behbod F, Alfaro MP, Buchholz TA and Rosen JM: WNT/beta-catenin mediates radiation resistance of mouse mammary progenitor cells. Proc Natl Acad Sci USA 104: 618-623, 2007.

58. Wu Y, Ginther C, Kim J, Mosher N, Chung S, Slamon D and Vadgama JV: Expression of Wnt 3 activates Wnt $/ \beta$-catenin pathway and promotes EMT-like phenotype in trastuzumab-resistant HER2-overexpressing breast cancer cells. Mol Cancer Res 10: 1597-1606, 2012.

59. Ahmad A, Sarkar SH, Bitar B, Ali S, Aboukameel A, Sethi S, Li Y, Bao B, Kong D, Banerjee S, et al: Garcinol regulates EMT and Wnt signaling pathways in vitro and in vivo, leading to anticancer activity against breast cancer cells. Mol Cancer Ther 11: 2193-2201, 2012.

60. Zhao Z, Lu P, Zhang H, Xu H, Gao N, Li M and Liu C: Nestin positively regulates the $\mathrm{Wnt} / \beta$-catenin pathway and the proliferation, survival and invasiveness of breast cancer stem cells. Breast Cancer Res 16: 408, 2014

61. Mukherjee N, Bhattacharya N, Alam N, Roy A, Roychoudhury S and Panda CK: Subtype-specific alterations of the Wnt signaling pathway in breast cancer: clinical and prognostic significance. Cancer Sci 103: 210-220, 2012

62. Xiang T, Li L, Yin X, Zhong L, Peng W, Qiu Z, Ren G and Tao Q: Epigenetic silencing of the WNT antagonist Dickkopf 3 disrupts normal $\mathrm{Wnt} / \beta$-catenin signalling and apoptosis regulation in breast cancer cells. J Cell Mol Med 17: 1236-1246, 2013. 